\title{
Reliability assessment considering stress drift and shock damage caused by stress transition shocks in a dynamic environment
}

\author{
HUANG Tingting, PENG Bo*, ZHAO Yuepu, and YU Zixuan
}

School of Reliability and Systems Engineering, Beihang University, Beijing 100191, China

\begin{abstract}
Products are often subject to dynamic environmental conditions in field use. When stress transition occurs, products may be exposed to instantaneous shocks that result in shock damages to the products, causing a permanent change of the degradation signals. Meanwhile, under some conditions, instantaneous shocks also lead to stress drift, causing a temporary change of the degradation signals. In this paper, a degradation model is proposed to assess the reliability and predict the residual lifetime of products operating in a dynamic environment considering shock damage and stress drift. The model is established based on a Wiener process which combines a stress-dependent degradation rate function, a shock damage function and a stress drift function in response to the dynamic environment. The shock damage function is established as a linear function of the stress transition start level and the stress level increment. The stress drift function is established as the difference value of a specified function at the stress transition start and end levels. A simulation study is presented to demonstrate the application of the model, and a case study for miniature light bulbs is used to validate the effectiveness of the proposed model.
\end{abstract}

Keywords: degradation modeling, dynamic environment, stress drift, shock damage, Wiener process.

DOI: $10.21629 / J S E E .2019 .05 .18$

\section{Introduction}

Many products experience complex environment (stress and load) in their life cycle, and the environment may change dynamically. Dynamic environments can be categorized as two types: with and without shocks. When shocks are considered, they are differentiated as random shocks and stress transition shocks. Under some conditions, when the stress transition shock occurs, the

Manuscript received July 03, 2018

*Corresponding author.

This work was supported by the National Natural Science Foundation of China (NSFC71601009) and the Technical Foundation Program from the Ministry of Industry and Information Technology of China (JSZL2015601B010). instantaneous shock on products causes shock damage and stress drift to products at the same time. The corresponding change on the degradation signal can be divided into two parts: permanent and temporary. The permanent part is shock damage. The temporary part is stress drift, i.e., when the stress level changes instantaneously, the degradation signal changes correspondingly; but when the stress level changes back, the degradation signal also returns to its previous state. Relevant literature in the corresponding areas is discussed as follows.

Degradation models considering stationary environmental conditions are studied extensively in the literature. In these models, degradation processes are characterized by using stochastic processes, e.g., Wiener processes, gamma processes, and Levy processes. The advantage of the Wiener process is that the distribution of the first passage time (FPT) can be formulated analytically, known as the inverse Gaussian distribution. Si et al. [1] proposed a degradation model based on the Wiener process to predict the remaining useful life (RUL) of products, and a recursive filter algorithm and a Bayesian method were used to estimate the distribution of the RUL. Wang [2] utilized the Wiener process to model real-time degradation signals and computed the residual lifetime distribution (RLD) of a single component. Bridge beam data is used to fit the proposed model, and the bootstrap method is also used for assessing the uncertainties of the estimators. Wang et al. [3] proposed a degradation model based on a generalized Wiener degradation process considering the nonlinearity, the temporal uncertainty, and the product-to-product variability of the degradation process. The Gamma process is a stochastic process with independent and non-negative increments with a gamma distribution and an identical scale parameter [4], and it is suitable to model degradation processes that are monotonic. Lawless et al. [5] proposed a model based on a gamma process with random 
effect across units to characterize the degradation process which is monotonic with conditionally independent increments. A Levy process is a continuous time stochastic process with independent and stationary increments and with right continuous sample paths having left limits. It is composed of a deterministic part, a Wiener process and an integral of compound poisson processes. Shu et al. [6] modeled the degradation processes with random jumps and random sizes based on Levy subordinators and nonGaussian Ornstein-Uhlenbeck processes.

Products may experience time-varying environment in their life cycle. For example, bearings in rotating machinery may perform under different working conditions, and the bearings may suffer dynamic working loads and speeds [7]. In addition, for a cylinder in an engine system, the seal ring of the cylinder may also be subject to a time-varying pressure when the engine system is loading [8]. Thus, it is not reasonable to assume that environmental conditions remain constant in degradation modeling for such products. Some work have been done on degradation modeling considering dynamic environments without shocks. Liao et al. [9] proposed a degradation model to predict the RUL of individual components under time-varying operating conditions through a Bayesian technique. $\mathrm{Xu}$ et al. [10] proposed a class of general path degradation models with dynamic covariates to characterize the random effects in a degradation process. Gebraeel et al. [11] presented a degradation model to compute the RLD of components operating in a dynamic environment based on real-time dynamic environmental information, insitu degradation signals, and degradation characteristics of components population. Bian et al. [12] established a degradation model based on a Wiener process with the stress-dependent degradation rate function in response to a continuous dynamic environment. Historical and realtime data are used to update the degradation model and RLD based on a Bayesian framework. A case study for ball bearings under multiple continuously varying environmental conditions (loads and rotational speeds) is provided. Zhai et al. [13] proposed a degradation model based on a Wiener process considering the dynamic block effect, which is defined as the temporal correlations in the degradation among test subjects in the same test rack under the common dynamic environments. Lumen maintenance data of blue light-emitting diodes is used to verify the effectiveness of the proposed model. Flory et al. [14] proposed a stochastic modeling framework based on a Wiener process to approximate the degradation process for estimating the lifetime of products, when the randomly-varying environment conditions are not known, or cannot be easily discerned. Jin et al. [15] proposed a degradation model and lifetime prediction method of the momentum wheel considering the dynamic bearing temperature based on a Wiener process with drift. Huang et al. [16] proposed a method to model the non-linear degradation behaviors of LED devices considering dynamic and random variations. Zhai et al. [17] proposed a degradation model with adaptive drift in a dynamic field environment. In this model, the adaptive drift is modeled based on a Wiener process. Hong et al. [18] proposed a general path degradation model with individual random effects to predict the lifetime of an organic coating in a dynamic environment. Peng et al. [19] proposed parametric inverse Gaussian process models with Bayesian framework to analyze the degradation process of products in dynamic situations. In this model, the time-varying degradation rates are incorporated to describe effects of dynamic situations on products, and random effects are used to model the unit-to-unit variability of products. Li et al. [20] presented a method to predict the RUL of systems in dynamic operational conditions and condition-specific failure zones. In this model, the degradation rate is affected by dynamic environments which are assumed to be expressed as a discrete-time Markov chain (DTMC). The failure thresholds are also determined by the environments and described as different failure zones. Liu et al. [21] proposed a degradation model based on a Wiener process to estimate the lifetime of the systems under dynamic conditions. The drift parameter and diffusion parameter are used to describe the variation of the individuals and the degradation property of the whole population respectively. The proposed model is applied to the Lithium-ion battery devices under three levels of charging and discharging rates.

In some engineering applications, products may be subject to dynamic environments with shocks. For these cases, effects of shocks should be considered in degradation modeling. Shocks are differentiated as random shocks and stress transition shocks. Random shocks occur at unfixed times. Commonly discussed random shocks are extreme shock, cumulative shock, $\delta$-shock, and run shock. Rafiee et al. [22] proposed a reliability model considering two dependent failure processes of soft failure due to degradation and hard failure due to shocks. In the model, the hard failure threshold may change based on the change of degradation. Some research work discussed a mix of different types of shocks. Hao et al. [23] proposed a degradation model for mutually dependent competing failure processes due to degradation and random shocks. Random shocks considered in this model are extreme shocks and cumulative shocks. Rafiee et al. [24] proposed a degradation model for dependent competing failure processes of degradation and random shocks. Shock patterns considered in this model are generalized extreme shock, generalized $\delta$-shock, generalized $m$-shock and generalized run shock. 
Hao et al. [25] proposed a degradation model for a degradation process with soft and hard failures considering the effect of the generalized extreme shock and the generalized $\delta$-shock on the thresholds of the shocks. Rafiee et al. [26] proposed a generalized mixed shock model considering the extreme shock, $\delta$-shock, and run shock. On the other hand, stress transition shocks occur at fixed times, i.e., the stress level changes instantaneously. Bian et al. [27] considered that stress transitions may induce the shock damage to products at stress transition times. The shock damage is assumed to have a linear correlation with stress increment. A thrust ball bearing degradation data under dynamic load and speed conditions is analyzed by using the proposed model.

Under some conditions, stress transition shocks also lead to stress drift. Unlike the shock damage, the stress drift may not induce any damage to products, but the degradation signal of the product changes correspondingly. Thus, it is necessary to compensate the stress drift to improve the accuracy of the prediction of product lifetime and reliability. Some work has been done on the stress drift compensation for the purpose of output compensation based on the software compensation method. Wang et al. [28] discussed the effects of temperature change on the output of a hemispherical resonator gyroscope (HRG), and proposed a temperature drift compensation model for HRG based on the relationship between the temperature and the natural frequency of the resonator. $\mathrm{Xu}$ et al. applied support vector machine (SVM) to compensate for the effects of temperature drift on the output of a dynamically tuned gyroscope (DTG), combined with empirical mode decomposition (EMD) [29] and wavelet transform (WT) [30] to eliminate the noises. Hong [31] proposed a nonlinear fuzzy model to compensate the temperature dependent non-linear characteristics of zero-rate-output bias drift of the resonant rate sensor (RRS). Wang et al. [32] proposed a pre-processing method to compensate temperature drift of super-luminescent diode (SLD) in the accelerated degradation testing (ADT) to improve the accuracy of the lifetime prediction. SVMs and the filtering method based on Wavelet analysis are used to compensate the stress drift. In addition, a hardware compensation method is also presented in this field. Baras et al. [33] proposed a compensation structure which is dependent on resonators to compensate the temperature drift of a hybrid low temperature co-fired ceramics (LTCC) oscillator.

A degradation process with the stress drift phenomenon shows a reversible characteristic, and this is against the assumption of most existing degradation models which assume that the degradation process changes monotonously or has random variations around a monotonic mean degradation level. Thus, without considering stress drift, the ex- isting models are not applicable. For the works that have discussed stress drift, it is assumed that the stress drift effect is known in advance. It is either acquired based on engineering experience or by operating a stress drift test to obtain an accurate stress drift vs. stress level relationship, and then compensate the stress drift effect for data in further tests. However, such tests require a large amount of products, and for many cases, it is impossible to perform a stress drift test in advance due to limited products considering cost, which makes such methods not applicable.

A degradation model based on a Wiener process combining with a stress-dependent degradation rate function, a shock damage function, and a stress drift function is proposed to characterize the degradation process of a product operating in a dynamic environment. It considers both the shock damage and stress drift effects of stress transition shocks. In addition, it relaxes the assumption of prespecified stress drift effect.

The remainder of the paper is organized as follows. Section 2 describes the degradation model in a dynamic environment with shock damage and stress drift. In Section 3, a simulation study is presented for the demonstration of this model. A case study of miniature light bulbs is illustrated to verify the effectiveness of this model in Section 4. Section 5 summarizes the work.

\section{Degradation modeling in dynamic environments considering stress drift}

\subsection{Model description}

For any time $t(t \geqslant 0)$, let $X(t)$ be the degradation signal of the product at time $t$, and $w(t)$ denotes the environmental state at time $t . J_{1}\left(w\left(\tau_{i}\right)\right)$ represents the change of the degradation signal due to the shock damage caused by the $i$ th instantaneous shock at time $\tau_{i}$, and $J_{2}\left(w\left(\tau_{i}\right)\right)$ denotes the change of the degradation signal due to the stress drift caused by the $i$ th instantaneous shock at time $\tau_{i}$. It is assumed that the shock damage only occurs when the stress level increases instantaneously, but does not occur when the stress level decreases, which is consistent with many industrial applications. This assumption can be relaxed if required.

Changes of the degradation signal due to the shock damage with and without stress drift are illustrated in Fig. 1. In Fig. 1(a), when the stress level increases instantaneously at time $\tau_{1}$, only the shock damage occurs, and results in an increment of degradation signal $J_{1}\left(w\left(\tau_{1}\right)\right)$. In Fig. 1(b), when the stress level increases instantaneously at time $\tau_{1}$, both shock damage and stress drift occur at the same time, and result in increments of the degradation signal, $J_{1}\left(w\left(\tau_{1}\right)\right)$ and $J_{2}\left(w\left(\tau_{1}\right)\right)$ respectively. Unlike the shock damage, the stress drift does not induce any damage to 
the product, and the increment of the degradation signal caused by the stress drift is temporary. Thus, when the stress level changes back to its initial level at time $\tau_{2}$, the degradation signal has a decrement for the same amount, i.e., $J_{2}\left(w\left(\tau_{1}\right)\right)=-J_{2}\left(w\left(\tau_{2}\right)\right)$. Note that, the stress level does not have to change back to its start level, but it is assumed so in this paper, and it can be later extended to more general cases.

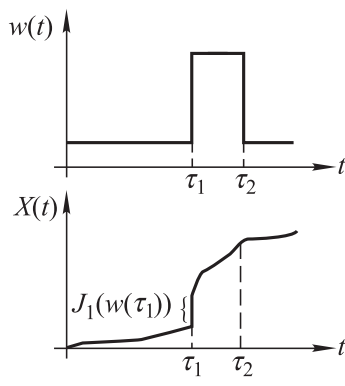

(a) Shock damage only

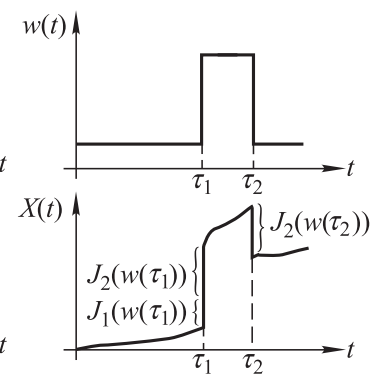

(b) Shock damage with stress drift
Fig. 1 Shock damage and stress drift

When the stress level decreases instantaneously, it is assumed that there is no shock damage, and thus no increment of the degradation signal; when the stress level increases instantaneously, the increment of the degradation signal caused by the shock damage is assumed as a linear function of the stress level increment and stress transition start level based on engineering experience. Thus, the shock damage function can be expressed as follows.

If the stress level decreases instantaneously at time $\tau_{i}$,

$$
J_{1}\left(w\left(\tau_{i}\right)\right)=0
$$

If the stress level increases instantaneously at time $\tau_{i}$,

$$
J_{1}\left(w\left(\tau_{i}\right)\right)=\alpha \Delta w\left(\tau_{i}\right)+\beta w\left(\tau_{i}^{-}\right)
$$

where $\alpha$ and $\beta$ are parameters to be estimated, $\Delta w\left(\tau_{i}\right)$ is the increment of the stress level at time $\tau_{i}$, and $w\left(\tau_{i}^{-}\right)$denotes the stress transition start level just prior to time $\tau_{i}$.

The stress drift function may take different forms for various applications. When the stress type is voltage, the degradation signal usually shows a power law relationship with the stress level, as shown in Fig. 2. In Fig. 2, $w_{i}(i=1,2,3,4)$ denote four different stress levels, $w_{4}>$ $w_{3}>w_{2}>w_{1}$, and $X_{w_{i}}(0)(i=1,2,3,4)$ are initial degradation signals under corresponding stress level $w_{i}$. Thus, considering voltage as an example, the stress drift function can be specified as difference values of power law functions at the stress transition end and start levels,

$$
J_{2}\left(w\left(\tau_{i}\right)\right)=\lambda\left(w\left(\tau_{i}^{+}\right)\right)^{\gamma}-\lambda\left(w\left(\tau_{i}^{-}\right)\right)^{\gamma}
$$

where $\gamma$ and $\lambda$ are parameters to be estimated, and $w\left(\tau_{i}^{+}\right)$ is the stress transition end level at time $\tau_{i}$.

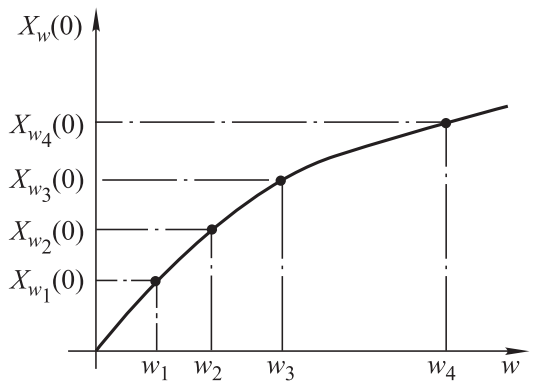

Fig. 2 Relationship between degradation signal and stress level

The degradation signal $X(t)$ for a product operating in a dynamic environment considering shock damage and stress drift can be expressed as

$$
\begin{aligned}
X(t)= & X(0)+\int_{0}^{t} r(w(v)) \mathrm{d} v+\sigma B(t)+ \\
& \sum_{i=1}^{n(t)}\left[J_{1}\left(w\left(\tau_{i}\right)\right)+J_{2}\left(w\left(\tau_{i}\right)\right)\right]
\end{aligned}
$$

where $X(0)$ is the initial degradation signal, $r(w(t))$ denotes the degradation rate of the product at time $t$ under the stress level $w(t), B(t)$ is a standard Wiener process, $\sigma$ is the diffusion parameter of the Wiener process, $B(t) \sim N(0, t)$, and $n(t)$ is the number of stress transition shocks occurring up to time $t$.

\subsection{Parameter estimation}

Assume that the stress profile has discrete increments when the stress level changes, the degradation model can then be modified as

$$
\begin{aligned}
X(t) \approx & X(0)+\sum_{j=1}^{m(t)} r\left(w\left(t_{j}\right)\right) \Delta t_{j}+\sigma B(t)+ \\
& \sum_{i=1}^{n(t)}\left[J_{1}\left(w\left(\tau_{i}\right)\right)+J_{2}\left(w\left(\tau_{i}\right)\right)\right]
\end{aligned}
$$

where $m(t)$ is the total number of degradation measurements until time $t, t_{j}$ is the time of the $j$ th degradation measurement, and $\Delta t_{j}$ is the time interval between the $(j-1)$ th and the $j$ th degradation signals (time interval between two successive measurements is assumed to be a constant in this paper).

The nonlinear least-square method is used to estimate the parameters $\alpha, \beta, \gamma$ and $\lambda$ in shock damage and stress drift functions. Let $n$ denote the number of instantaneous shocks observed based on the stress profile and data obtained for parameter estimation, i.e., $n=n(t)$ while $t$ is the test time when the last degradation measurement is obtained. The residual sum of squares equation with the parameters $\alpha, \beta, \gamma$, and $\lambda$ is denoted by 


$$
\begin{gathered}
Q(\alpha, \beta, \gamma, \lambda)=\sum_{i=1}^{n}\left[J_{1}\left(w\left(\tau_{i}\right)\right)+J_{2}\left(w\left(\tau_{i}\right)\right)-\alpha \Delta w\left(\tau_{i}\right)-\right. \\
\left.\beta w\left(\tau_{i}^{-}\right)-\lambda\left(w\left(\tau_{i}^{+}\right)\right)^{\gamma}+\lambda\left(w\left(\tau_{i}^{-}\right)\right)^{\gamma}\right]^{2}
\end{gathered}
$$

and

$$
Q(\widehat{\alpha}, \widehat{\beta}, \widehat{\gamma}, \widehat{\lambda})=\min Q(\alpha, \beta, \gamma, \lambda) .
$$

By taking the first derivative of the residual sum of the squares equation with respect to the parameters, and setting them to zero, estimates $\widehat{\alpha}, \widehat{\beta}, \widehat{\gamma}$ and $\widehat{\lambda}$ can be obtained by solving (6).

$$
\left\{\begin{array}{c}
\frac{\partial Q(\alpha, \beta, \gamma, \lambda)}{\partial \alpha}=-2 \sum_{i=1}^{n}\left[J_{1}\left(w\left(\tau_{i}\right)\right)+J_{2}\left(w\left(\tau_{i}\right)\right)-\right. \\
\alpha \Delta w\left(\tau_{i}\right)-\beta w\left(\tau_{i}^{-}\right)- \\
\left.\lambda\left(w\left(\tau_{i}^{+}\right)\right)^{\gamma}+\lambda\left(w\left(\tau_{i}^{-}\right)\right)^{\gamma}\right] \Delta w\left(\tau_{i}\right)=0 \\
\frac{\partial Q(\alpha, \beta, \gamma, \lambda)}{\partial \beta}=-2 \sum_{i=1}^{n}\left[J_{1}\left(w\left(\tau_{i}\right)\right)+J_{2}\left(w\left(\tau_{i}\right)\right)-\right. \\
\alpha \Delta w\left(\tau_{i}\right)-\beta w\left(\tau_{i}^{-}\right)- \\
\left.\lambda\left(w\left(\tau_{i}^{+}\right)\right)^{\gamma}+\lambda\left(w\left(\tau_{i}^{-}\right)\right)^{\gamma}\right] w\left(\tau_{i}^{-}\right)=0 \\
\frac{\partial Q(\alpha, \beta, \gamma, \lambda)}{\partial \gamma}=-2 \sum_{i=1}^{n}\left[J_{1}\left(w\left(\tau_{i}\right)\right)+J_{2}\left(w\left(\tau_{i}\right)\right)-\right. \\
\alpha \Delta w\left(\tau_{i}\right)-\beta w\left(\tau_{i}^{-}\right)-\lambda\left(w\left(\tau_{i}^{+}\right)\right)^{\gamma}+ \\
\left.\lambda\left(w\left(\tau_{i}^{-}\right)\right)^{\gamma}\right]\left[\lambda\left(w\left(\tau_{i}^{+}\right)\right)^{\gamma} \ln \left(w\left(\tau_{i}^{+}\right)\right)-\right. \\
\left.\lambda\left(w\left(\tau_{i}^{-}\right)\right)^{\gamma} \ln \left(w\left(\tau_{i}^{-}\right)\right)\right]=0 \\
\frac{\partial Q(\alpha, \beta, \gamma, \lambda)}{\partial \lambda}=-2 \sum_{i=1}^{n}\left[J_{1}\left(w\left(\tau_{i}\right)\right)+J_{2}\left(w\left(\tau_{i}\right)\right)-\right. \\
\alpha \Delta w\left(\tau_{i}\right)-\beta w\left(\tau_{i}^{-}\right)-\lambda\left(w\left(\tau_{i}^{+}\right)\right)^{\gamma}+ \\
\left.\lambda\left(w\left(\tau_{i}^{-}\right)\right)^{\gamma}\right]\left[\left(w\left(\tau_{i}^{+}\right)\right)^{\gamma}-\left(w\left(\tau_{i}^{-}\right)\right)^{\gamma}\right]=0
\end{array}\right.
$$

The degradation rate function may depend on the various environmental or load factors. Considering voltage as the stress type for example, based on engineering experience, the degradation rate has a power law form, $r(w(t))=$ $a(w(t))^{b}$, where $a$ and $b$ are parameters to be estimated. To reduce the complexity of calculation, the effects of shock damages and stress drifts are deducted from degradation signal measurements. Thus, the degradation signal $X(t)$ can be redefined as

$$
H(t)=X(t)-\sum_{i=1}^{n(t)}\left[J_{1}\left(w\left(\tau_{i}\right)\right)+J_{2}\left(w\left(\tau_{i}\right)\right)\right]
$$

and

$$
H(t) \approx X(0)+\sum_{j=1}^{m(t)} a\left(w\left(t_{j}\right)\right)^{b} \Delta t_{j}+\sigma B(t) .
$$

Let $m$ denote the total number of the degradation signal measurement obtained for parameter estimation, i.e., $m=m(t)$ while $t$ is the test time when the last degradation measurement is obtained. Based on the independent increment property of a Wiener process, $\Delta H\left(t_{j}\right) \sim$ $N\left(a\left(w\left(t_{j}\right)\right)^{b} \Delta t_{j}, \sigma^{2} \Delta t_{j}\right)(j=1,2, \ldots, m)$, and the likelihood function with parameters $a, b$, and $\sigma$ is denoted by

$$
\begin{gathered}
L(a, b, \sigma)=\prod_{j=1}^{m} \frac{1}{\sigma \sqrt{2 \pi \Delta t_{j}}} . \\
\exp \left[-\frac{\left(\Delta H\left(t_{j}\right)-a\left(w\left(t_{j}\right)\right)^{b} \Delta t_{j}\right)^{2}}{2 \sigma^{2} \Delta t_{j}}\right] .
\end{gathered}
$$

The log likelihood function is

$$
\begin{gathered}
l(a, b, \sigma)=-\frac{1}{2} \sum_{j=1}^{m} \ln \left(2 \pi \Delta t_{j}\right)-\frac{1}{2} m \ln \sigma^{2}- \\
\sum_{j=1}^{m}\left[\frac{\left(\Delta H\left(t_{j}\right)-a\left(w\left(t_{j}\right)\right)^{b} \Delta t_{j}\right)^{2}}{2 \sigma^{2} \Delta t_{j}}\right] .
\end{gathered}
$$

Estimates of parameters $a, b$ and $\sigma$ can be obtained by maximizing the log-likelihood function, as in (10).

\subsection{Residual lifetime prediction}

The FPT $T$ denotes the first time that the degradation signal $\{X(t): t \geqslant 0\}$ crosses a fixed threshold $D$.

$$
T=\inf \{t>0: X(t) \geqslant D\}
$$

Reliability at time $t$ can be expressed as

$$
R(t)=P\{T>t\}=P\{\max X(u)<D, 0 \leqslant u \leqslant t\} .
$$

In a degradation process, the effect of stress drift results in increments of the degradation signal that does not correspond to real damage to products. Thus, the increments of the degradation signal described by the stress drift function should be eliminated from the degradation signal, and results in $Y(t)$.

$$
Y(t)=X(t)-\sum_{i=1}^{n(t)} J_{2}\left(w\left(\tau_{i}\right)\right)
$$

The redefined degradation signal $Y(t)$ can be decomposed into deterministic and stochastic components

$$
Y(t)=\zeta(t)+\sigma B(t)
$$

where

$$
\zeta(t)=Y(0)+\int_{0}^{t} r(w(v)) \mathrm{d} v+\sum_{i=1}^{n(t)} J_{1}\left(w\left(\tau_{i}\right)\right) .
$$

$\zeta(t)$ is the deterministic component, and $\sigma B(t)$ is the stochastic component. $Y(0)$ is the initial value of the redefined degradation signal. 
Then, the reliability function is expressed as

$$
\begin{gathered}
R(t)=P\{\max Y(u)<D, 0 \leqslant u \leqslant t\}= \\
P\left\{B(u)<\frac{D-\zeta(u)}{\sigma}, 0 \leqslant u \leqslant t\right\}= \\
P\{B(u)<g(u), 0 \leqslant u \leqslant t\} \\
g(t)=\frac{D-\zeta(t)}{\sigma}
\end{gathered}
$$

where $g(t)$ represents the time-dependent boundary according to a standard Wiener process $B(t)$.

$g(t)$ is a curved boundary, and thus, needs to be linearized to obtain approximation solutions [34]. According to Daniels [35], when the crossing boundary is linear, $f(t)$ can be approximated by using the probability density function (PDF) of FPT.

$$
\begin{gathered}
f(t) \approx \frac{1}{\sqrt{2 \pi t}}\left(\frac{D-\zeta(t)+r(w(t)) t}{t \sigma}\right) . \\
\exp \left[-\frac{(D-\zeta(t))^{2}}{2 t \sigma^{2}}\right]
\end{gathered}
$$

The reliability function is expressed as follows:

$$
R(t)=1-\int_{0}^{t} f(v) \mathrm{d} v .
$$

\section{Simulation study}

\subsection{Data generation}

A stress profile with discrete increments is used for the simulation study. Fig. 3 shows the stress profile. A power law model $r(w(t))=a(w(t))^{b}$ is used to describe the degradation rate function. The initial degradation signal $X(0)=0$, and it is assumed that a population of identical components degrade from the same initial value of the degradation signal. Parameter settings are listed in Table 1.

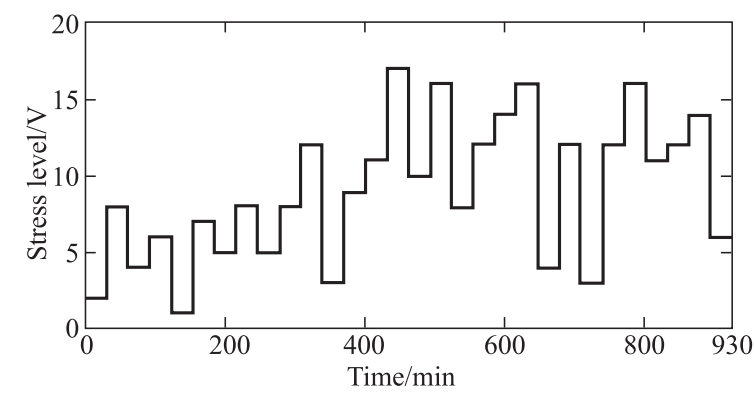

Fig. 3 Stress profile in the simulation study

Table 1 Parameter settings

\begin{tabular}{cccccccc}
\hline$\alpha$ & $\beta$ & $\gamma$ & $\lambda$ & $a$ & $b$ & $\sigma$ & $D$ \\
\hline 9 & 3 & 3 & 2.5 & 0.1 & 1.5 & 2.8 & 2000 \\
\hline
\end{tabular}

In total 100 paths (100 products) of degradation signals are generated every $0.01 \mathrm{~min}$ based on the proposed model in Section 2, as shown in Fig. 4. The first 45000 data before (and include) $450 \mathrm{~min}$ are used to fit the model, and the last 48000 data from 45001 to 93000 , i.e., $450 \mathrm{~min}$ (not include) to $930 \mathrm{~min}$, are used to test the accuracy of the estimates.

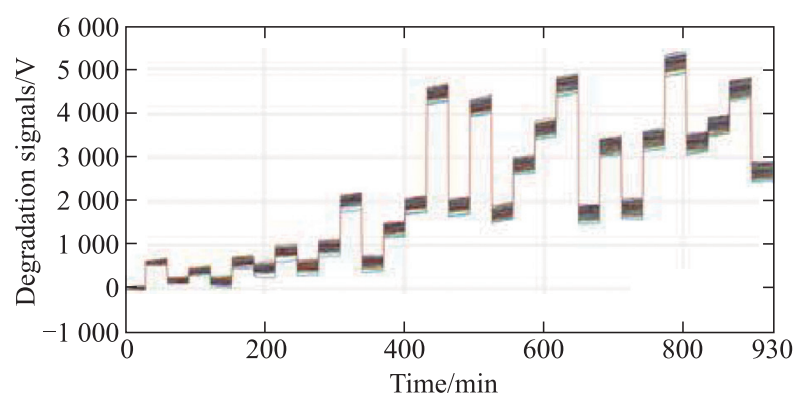

Fig. 4 Degradation signals for 100 products

\subsection{Failure time calculation}

The increments of the degradation signal described by the stress drift function are eliminated from the degradation signal. Redefined degradation signals and the threshold are shown in Fig. 5. Failure times that the redefined degradation signals exceed the threshold are collected and listed in Fig. 6.

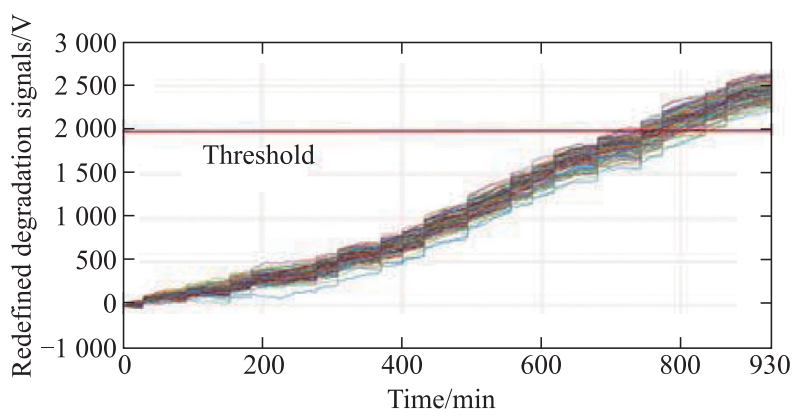

Fig. 5 Redefined degradation signals and the threshold

\begin{tabular}{|cccccccc|}
\hline \multicolumn{7}{c|}{ Failure time/min } \\
\hline 709 & 710 & 711 & 717 & 720 & 723 & 724 & 725 \\
726 & 728 & 729 & 729 & 731 & 731 & 733 & 734 \\
735 & 735 & 736 & 737 & 738 & 743 & 743 & 746 \\
747 & 756 & 757 & 758 & 758 & 759 & 760 & 761 \\
762 & 763 & 764 & 765 & 766 & 767 & 768 & 769 \\
772 & 773 & 773 & 774 & 775 & 776 & 777 & 777 \\
778 & 779 & 780 & 782 & 785 & 786 & 786 & 787 \\
787 & 791 & 793 & 796 & 802 & 803 & 804 & 804 \\
804 & 805 & 805 & 806 & 806 & 807 & 807 & 808 \\
809 & 810 & 811 & 812 & 812 & 813 & 813 & 817 \\
819 & 820 & 821 & 821 & 822 & 822 & 822 & 824 \\
826 & 828 & 828 & 829 & 830 & 831 & 833 & 834 \\
836 & 838 & 839 & 840 & - & - & - & - \\
\hline
\end{tabular}

Fig. 6 Failure time of 100 products 


\subsection{Parameter estimation}

Parameters $\alpha, \beta, \gamma$ and $\lambda$ in the shock damage function and stress drift function are calculated by using the least-square method. Based on the independent increment property of the Wiener process, parameters $a, b$ in the degradation rate function and the diffusion parameter $\sigma$ are estimated by using the maximum likelihood method. Estimates of the parameters are listed in Table 2.

Table 2 Estimates of parameters

\begin{tabular}{ccccccc}
\hline$\alpha$ & $\beta$ & $\gamma$ & $\lambda$ & $a$ & $b$ & $\sigma$ \\
\hline 8.86 & 2.98 & 2.93 & 2.56 & 0.098 & 1.491 & 2.73 \\
\hline
\end{tabular}

\subsection{Validation of estimation of parameters}

Estimation of parameters follows a multi-stage approach, i.e., parameters $a, b$ and $\sigma$ are estimated after parameters $\alpha, \beta, \gamma$ and $\lambda$, and estimates of parameters $a, b$ and $\sigma$ are dependent on the estimates of parameters $\alpha, \beta, \gamma$ and $\lambda$. To validate the effectiveness of the estimation approach, simulation is operated for 1000 times based on the parameter settings as in Table 1. Degradation data are generated and used for parameter estimation. Bias, standard deviation (SD) and root mean squared errors (RMSE) are calculated based on the estimates of parameters, as listed in Table 3. Simulation results show that the accuracy and precision of the estimates of the parameters are acceptable.

Table 3 Estimation results for parameters based on 1000 times simulations estimates of parameters

\begin{tabular}{cccccccc}
\hline Method & $\alpha$ & $\beta$ & $\gamma$ & $\lambda$ & $a$ & 0 \\
\hline Bias & 0.0022 & -0.0065 & 0.0055 & 0.0036 & -0.0033 & 0.0024 & 0.0041 \\
SD & 0.0265 & 0.0423 & 0.0588 & 0.0467 & 0.0322 & 0.0455 & 0.0322 \\
RMSE & 0.0299 & 0.0442 & 0.0626 & 0.0452 & 0.0287 & 0.0421 \\
\hline
\end{tabular}

\subsection{Reliability prediction}

According to the estimates of the parameters, and future stress profile, reliability is estimated by using the proposed method from $450 \mathrm{~min}$ (not include) to $930 \mathrm{~min}$ (time unit 0.01 ) based on the future stress level at each time point. Reliability is also estimated by using the Kaplan-Meier (K-M) method based on failure time data for comparison purposes. Estimation results based on the proposed model (black curve) and the K-M method (red curve) are shown in Fig. 7. The RMSE of reliability predictions using the proposed model and the K-M method at failure time points is 0.0126 . For the purpose of demonstration, the estimation of reliability based on historical stress profile, historical degradation data, and future stress profile matches well with that of the K-M method based on the generated failure time data of the 100 products.

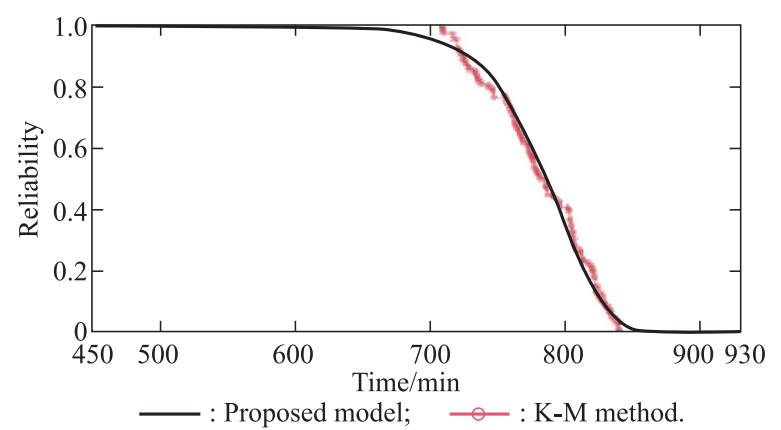

Fig. 7 Reliability estimates in the simulation study

\section{Case study}

\subsection{Degradation test system}

A degradation test system is established to obtain degra- dation data of miniature light bulbs operating in a dynamic environment. The system mainly consists of a programmable power supply, bulb circuit board, data acquisition board, compact data acquisition (DAQ) chassis, PC, data recording software, miniature light bulbs and resistors as shown in Fig. 8. The power supply provides voltage as the stress to the bulbs which are connected with resistors in series on the bulb circuit board [21].

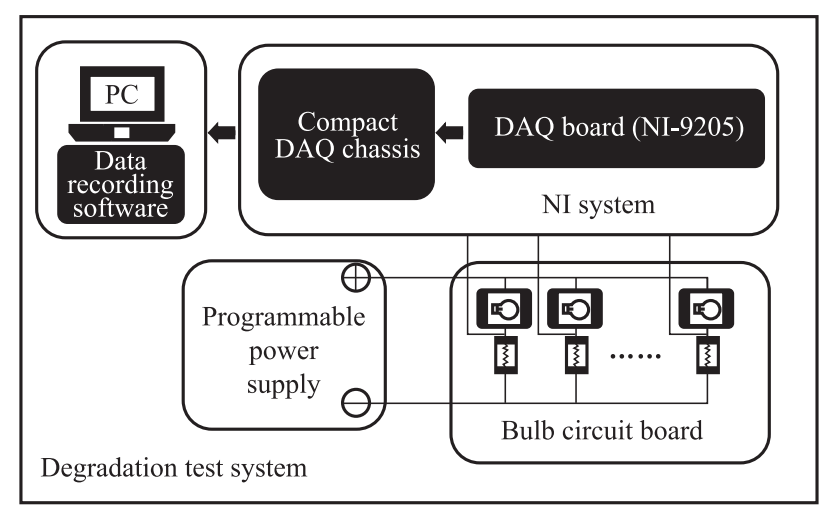

Fig. 8 Degradation test system for miniature bulbs [21]

\subsection{Degradation test}

Degradation tests are operated on 27 miniature bulbs in a dynamic environment. The stress profile is shown in Fig. 9. Voltages across the resistors are acquired every minute, and used as degradation signals that reflect the performance condition of the bulbs, and they drop down to a certain value near zero when the bulbs fail, as shown in Fig. 10. Fig. 11 shows the degradation signals of 27 bulbs from $410 \mathrm{~min}$ to $460 \mathrm{~min}$. Failure times of 27 bulbs are listed in Fig. 12. 


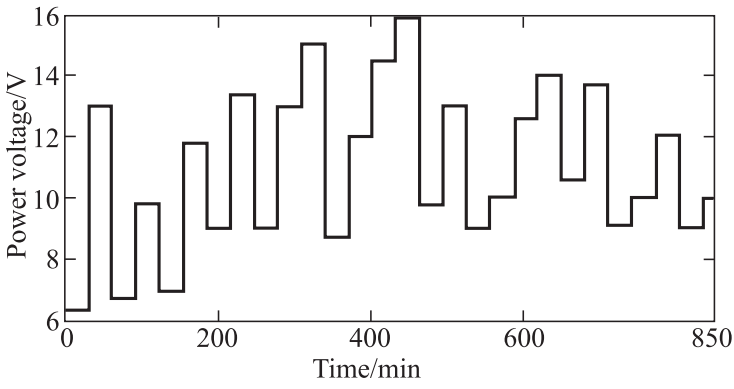

Fig. 9 Stress profile in the case study

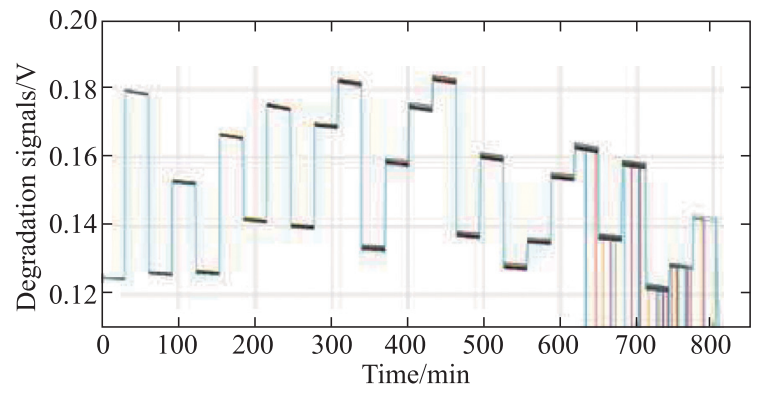

Fig. 10 Degradation signals for 27 miniature bulbs

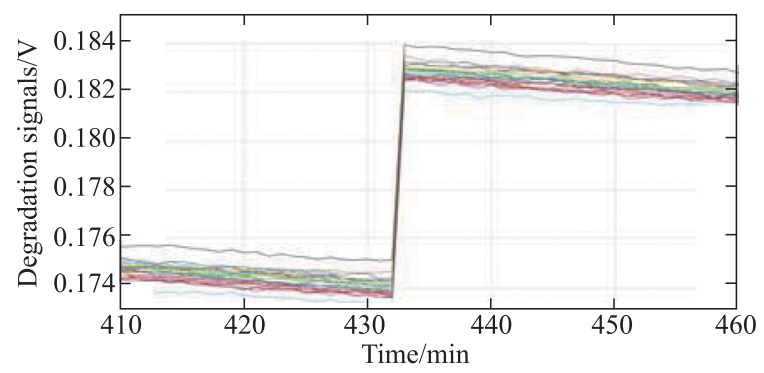

Fig. 11 Degradation signals from $410 \mathrm{~min}$ to $460 \mathrm{~min}$

\begin{tabular}{|lllllllll|}
\hline \multicolumn{7}{|c|}{ Failure time/min } \\
\hline 633 & 647 & 657 & 668 & 673 & 685 & 694 & 704 & 705 \\
716 & 727 & 730 & 735 & 741 & 743 & 746 & 752 & 754 \\
755 & 764 & 766 & 768 & 772 & 786 & 789 & 805 & 807 \\
\hline
\end{tabular}

Fig. 12 Failure time of 27 miniature bulbs

\subsection{Degradation percentage}

The initial degradation signal values of the 27 bulbs may be different due to individual variability. To assure the 27 bulbs have the same initial degradation signal value, degradation signal $X(t)$ is redefined as the degradation percentage, that is,

$$
X(t)=\frac{\text { initial signal }- \text { degradation signal }}{\text { initial signal }} .
$$

\subsection{Parameter estimation}

The first 450 data of each of the 27 bulbs before (and include) $450 \mathrm{~min}$ (in total 12150 data) are used to fit the model, and the rest are used to test the accuracy of the prediction. Parameters $\alpha, \beta, \gamma$ and $\lambda$ in the shock damage function and stress drift function, and $a, b$ in the degradation rate function, diffusion parameter $\sigma$, are estimated by using the proposed methods. The estimates of the parameters are listed in Table 4.

Table 4 Estimates of parameters

\begin{tabular}{ccccccc}
\hline$\alpha$ & $\beta$ & $\gamma$ & $\lambda$ & $a$ & $b$ & $\sigma$ \\
\hline 0.00343 & 0.00006 & 0.512 & 0.048 & $2.67 \times 10^{-5}$ & 1.98 & $0.2 \times 10^{-4}$ \\
\hline
\end{tabular}

Based on the estimates of the parameters, the increments of the degradation percentage described by the stress drift function are eliminated from the degradation percentage. Redefined degradation percentages of 27 bulbs and the threshold are shown in Fig. 13, while threshold $D$ is set as 0.24 based on historical experience.

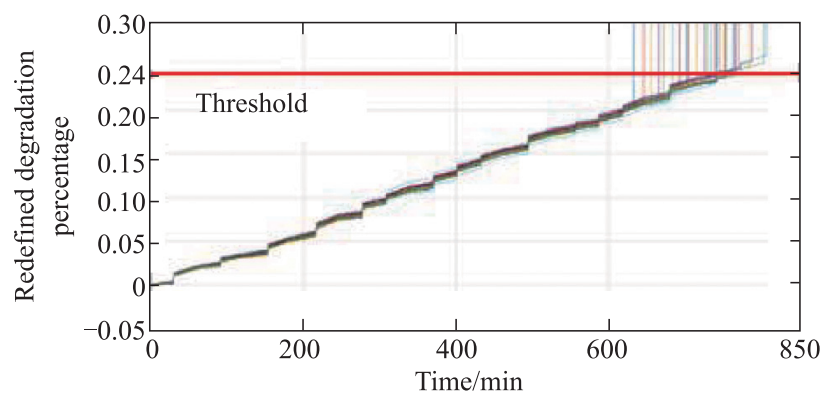

Fig. 13 Redefined degradation percentages and the threshold

\subsection{Reliability prediction and verification}

Reliability is estimated by using the proposed method at the time points from $451 \mathrm{~min}$ to $850 \mathrm{~min}$, based on the future stress level at each time point. Confidence intervals of bulbs reliability can be obtained based on the bootstrap method. In total 1000 sets of 27 censored degradation paths are sampled with replacement from the population, and reliability is estimated based on the corresponding data. The point-by-point reliability estimates of two-sided 95\% and $85 \%$ confidence intervals can then be obtained, and shown as the blue dash dot curve, and the green dash curve as in Fig. 14. Based on the results of calculation of confidence intervals of reliability, the medium lifetime intervals of miniature bulbs are $[722,744]$ ( $85 \%$ confidence intervals), and $[713,755]$ (95\% confidence intervals). The characteristic lifetime intervals are [736, 759] (85\% confidence intervals), and [727, 768] (95\% confidence intervals). In addition, reliability is also estimated by using the $\mathrm{K}-\mathrm{M}$ method based on the failure time data as listed in Fig. 12 for comparison. Estimation results are shown in Fig. 14. RMSE of reliability estimates of the proposed model and the K-M method at the failure time points is 0.0682 . The effectiveness of the proposed model is verified. 


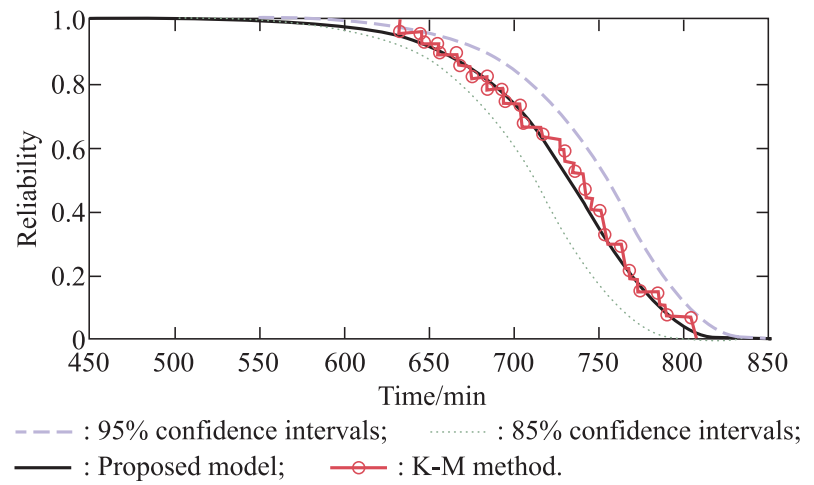

Fig. 14 Reliability estimates in the case study

\section{Conclusions}

In engineering applications, for some products, stress drift and shock damage may occur instantaneously at stress transition times in a time-varying environment. Existing stress drift compensation methods assume that the stress drift effect is known in advance by engineering experience or stress drift test. However, for some products with high cost or limited number of samples available, there is no empirical data, and it is impossible to perform the stress drift test. Thus, the relationship between stress drift and stress level cannot be obtained, and makes it impossible for degradation modeling and reliability prediction of the products.

A degradation model for products in a dynamic environment considering stress drift and shock damage is proposed. The model is established based on a stress drift function, a shock damage function, a time-dependent degradation rate function, and a Wiener process. Parameters are estimated by using the least-square method and maximum likelihood estimate. Residual lifetime prediction is based on future stress profile by using approximation of PDF of FPT by linearizing a curved boundary. A simulation study is presented for the demonstration of this model. A case study of miniature bulbs is used to verify the effectiveness of the proposed model. The proposed model relaxes the assumption of pre-specified stress drift effect. It can differentiate the effect of shock damage and stress drift caused by stress transition shocks based on historical data, and then eliminate the effects caused by stress drift in the prediction.

The work also raises a few important questions that require further research. First, for some practical problems, the stress drift function may be complex or has similar form as the shock damage function which makes it difficult to separate the effect of stress drift from that of shock damage. Second, only one type of stress is considered, and for many cases, products experience complex environment conditions (stress and load) in their life cycle. Third, it is assumed that the stress profile is deterministic and prespecified, and the assumption can be relaxed in the future research to consider a more complex case that the stress level changes randomly and the stress profile is unknown in advance.

\section{References}

[1] SI X, WANG W, HU C, et al. A Wiener-process-based degradation model with a recursive filter algorithm for remaining useful life estimation. Mechanical Systems \& Signal Processing, 2013: 35(1/2): 219-237.

[2] WANG X. Wiener processes with random effects for degradation data. Journal of Multivariate Analysis, 2010, 101(2): $340-351$.

[3] WANG X, BALAKRISHNAN N, GUO B. Residual life estimation based on a generalized Wiener degradation process. Reliability Engineering \& System Safety, 2014, 124: 13-23.

[4] NOORTWIJK J M. A survey of the application of gamma processes in maintenance. Reliability Engineering \& System Safety, 2009, 94(1): 2-21.

[5] LAWLESS J, CROWDER M. Covariates and random effects in a gamma process model with application to degradation and failure. Lifetime Data Analysis, 2004: 10(3): 213-227.

[6] SHU Y, FENG Q, KAO E P C. Levy-driven non-gaussian Ornstein-Uhlenbeck processes for degradation-based reliability analysis. AIIE Transactions, 2016, 48(11): 993-1003.

[7] XUE L, LI N, LEI Y. Incipient fault detection for rolling element bearings under varying speed conditions. Materials, 2017, 10(6): 675-684.

[8] LIAO B, SUN B, YAN M. Time-variant reliability analysis for rubber O-ring seal considering both material degradation and random load. Materials, 2017, 10(10): $1211-1220$.

[9] LIAO H, TIAN Z. A framework for predicting the remaining useful life of a single unit under time-varying operating conditions. IIE Transactions, 2013, 45(9): 964-980.

[10] XU Z, HONG Y, JIN R. Nonlinear general path models for degradation data with dynamic covariates. Applied Stochastic Models in Business and Industry, 2016, 32(2): 153-167.

[11] GEBRAEEL N, PAN J. Prognostic degradation models for computing and updating residual life distributions in a timevarying environment. IEEE Trans. on Reliability, 2008, 57(4): $539-550$.

[12] BIAN L, GEBRAEEL N. Stochastic methodology for prognostics under continuously varying environmental profiles. Statistical Analysis \& Data Mining, 2012, 6(3): 260-270.

[13] ZHAI Q Q, YE Z S. Degradation in common dynamic environments. Technometrics, 2017, 60(4): 461-471.

[14] FLORY J, KHAROUFEH J, GEBRAEEL N. A switching diffusion model for lifetime estimation in randomly varying environments. IIE Transactions, 2014, 46(11): 1227 - 1241.

[15] JIN G, MATTHEWS D, FAN Y, et al. Physics of failure-based degradation modeling and lifetime prediction of the momentum wheel in a dynamic covariate environment. Engineering Failure Analysis, 2013, 28(3): 222-240.

[16] HUANG J, GOLUBOVI D S, KOH S. Degradation modeling of mid-power white-light LEDs by using Wiener process. Optics Express, 2015, 23(15): A966.

[17] ZHAI Q, YE Z S. RUL prediction of deteriorating products using an adaptive wiener process model. IEEE Trans. on Industrial Informatics, 2017, PP(99): 1.

[18] HONG Y, DUAN Y, MEEKER W Q. Statistical methods for degradation data with dynamic covariates information and an 
application to outdoor weathering data. Technometrics, 2015, 47(3): $830-837$.

[19] PENG W, LI Y F, YANG Y J. Bayesian degradation analysis with inverse gaussian process models under time-varying degradation rates. IEEE Trans. on Reliability, 2017, 66(1): $84-96$.

[20] LI Q, GAO Z, TANG D. Remaining useful life estimation for deteriorating systems with time-varying operational conditions and condition-specific failure zones. Chinese Journal of Aeronautics, 2016, 29(3): 662-674.

[21] LIU T, SUN Q, FENG J. Residual life estimation under timevarying conditions based on a Wiener process. Journal of Statistical Computation and Simulation, 2017, 87(2): 211-226.

[22] RAFIEE K, FENG Q, COIT D W. Reliability analysis and condition-based maintenance for failure processes with degradation-dependent hard failure threshold. Quality and Reliability Engineering International, 2017, 33(7): 1351 - 1366.

[23] HAO S, YANG J, MA X. Reliability modeling for mutually dependent competing failure processes due to degradation and random shocks. Applied Mathematical Modelling, 2017: S0307904X17304067.

[24] RAFIEE K, FENG Q, COIT D W. Reliability modeling for dependent competing failure processes with changing degradation rate. IIE Transactions, 2014, 46(5): 483-496.

[25] HAO S, YANG J. Reliability analysis for dependent competing failure processes with changing degradation rate and hard failure threshold levels. Computers \& Industrial Engineering, 2018: S0360835218300871.

[26] RAFIEE K, FENG Q, COIT D W. Reliability assessment of competing risks with generalized mixed shock models. Reliability Engineering \& System Safety, 2017, 159: 1-11.

[27] BIAN L, GEBRAEEL N, KHAROUFEH J P. Degradation modeling for real-time estimation of residual lifetimes in dynamic environment. AIIE Transactions, 2015, 47(5): $471-$ 486.

[28] WANG X, WU W, FANG Z, et al. Temperature drift compensation for hemispherical resonator gyro based on natural frequency. Sensors, 2012, 12(5): 6434-6446.

[29] XU G P, TIAN W F, QIAN L. EMD and SVM based temperature drift modeling and compensation for a Dynamically Tuned Gyroscope (DTG). Mechanical Systems \& Signal Processing, 2007, 21(8): 3182-3188.

[30] XU G P, TIAN W F, JIN Z H, et al. Temperature drift modelling and compensation for a dynamically tuned gyroscope by combining WT and SVM method. Measurement Science \& Technology, 2007, 18(5): 1425-1432.

[31] HONG S. Compensation of nonlinear thermal bias drift of Resonant Rate Sensor using fuzzy logic. Sensors and Actuators A (Physical), 1999, 78(2-3): $143-148$.

[32] WANG L Z, JIANG T M, LI X Y, et al. A pre-processing method for degradation parameter. Proc. of the IEEE Prognostics and System Health Management Conference, 2012: 1-4.

[33] BARAS T, JACOB A F. Temperature drift compensation technique for a hybrid LTCC oscillator at 20GHz. Proc. of the European Microwave Integrated Circuits Conference, 2009:

$$
467-470 .
$$

[34] HUANG T, PENG B, COIT D W, et al. Degradation modeling and lifetime prediction considering effective shocks in a dynamic environment. IEEE Trans. on Reliability, 2019, 68(3): $819-830$.

[35] DANIELS H E. Approximating the first crossing-time density for a curved boundary. Bernoulli, 1996, 2(2): 133-143.

\section{Biographies}

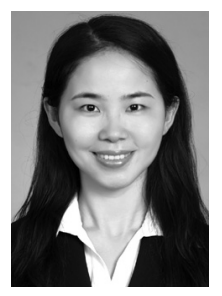

HUANG Tingting was born in 1981. She is an assistant professor for the School of Reliability and Systems Engineering, Beihang University, China. She worked as a postdoctoral for the Department of Industrial Engineering, Tsinghua University in 2011. She recieved her Ph.D. degree from the School of Reliability and Systems Engineering, Beihang University in 2010. She recieved her M.S. degree from the Department of Industrial and Systems Engineering, Virginia Tech in 2014. She was a visiting scholar in the Department of Industrial and Systems Engineering, Rutgers University, USA in 2008. Her research interests are accelerated life testing, accelerated degradation testing and other reliability and environment testing technology. Her recent work is on degradation modeling and reliability prediction of products in a dynamic environment considering shocks.

E-mail: htt@buaa.edu.cn

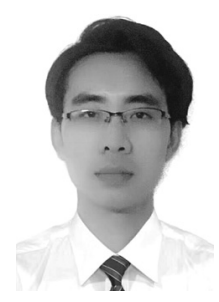

PENG Bo was born in 1986. He is currently working towards his Ph.D. degree in the School of Reliability and Systems Engineering, Beihang University. His research interests are degradation modeling and lifetime prediction for degradation tests. E-mail: pengb@buaa.edu.cn

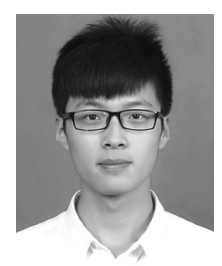

ZHAO Yuepu was born in 1996. He is currently working towards his Ph.D. degree in the School of Reliability and Systems Engineering, Beihang University. His research interests are degradation modeling and lifetime prediction for degradation tests. E-mail: yuepu_zhao@buaa.edu.cn

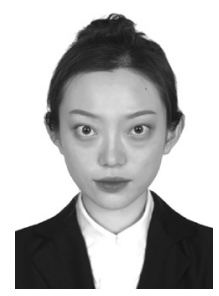

YU Zixuan was born in 1993. She is currently working towards her Ph.D. degree in the School of Reliability and Systems Engineering, Beihang University. Her research interests are multivariable degradation modeling and lifetime prediction. E-mail: yuzixx@buaa.edu.cn 